\title{
Vascular infarction by subcutaneous application of tissue factor targeted to tumor vessels with NGR-peptides: Activity and toxicity profile
}

\author{
JOHANNES DREISCHALÜCK ${ }^{1}$, CHRISTIAN SCHWÖPPE $^{1}$, TILMANN SPIEKER ${ }^{2}$, TORSTEN KESSLER ${ }^{1}$, \\ KLAUS TIEMANN $^{3}$, RUEDIGER LIERSCH ${ }^{1}, \mathrm{CHRISTOPH} \mathrm{SCHLIEMANN}^{1}$, MICHAEL KREUTER ${ }^{4}$, \\ ASTRID KOLKMEYER ${ }^{1}$, HEIKE HINTELMANN ${ }^{1}$, ROLF M. MESTERS ${ }^{1 *}$ and WOLFGANG E. BERDEL ${ }^{1 *}$ \\ ${ }^{1}$ Department of Medicine A, Hematology, Oncology and Pneumology, ${ }^{2}$ Gerhard Domagk Institute of Pathology, \\ ${ }^{3}$ Department of Medicine C, Cardiology, Angiology, University of Muenster, Albert-Schweitzer-Strasse 33, D-48129 \\ Muenster; ${ }^{4}$ Department of Medicine/Thoracic Oncology, Thoraxklinik at the University of Heidelberg, Heidelberg, Germany
}

Received July 16, 2010; Accepted September 2, 2010

DOI: 10.3892/ijo_00000790

\begin{abstract}
TF-NGR consists of the extracellular domain of the (truncated) tissue factor (tTF), a central molecule for coagulation in vivo, and the peptide GNGRAHA (NGR), a ligand of the surface protein aminopeptidase N (CD13). After deamidation of the NGR-peptide moiety, the fusion protein is also a ligand for integrin $\alpha_{v} \beta_{3}$ (CD51/CD61). Both surface proteins are upregulated on endothelial cells of tumor vessels. tTF-NGR showed binding to specific binding sites on endothelial cells in vitro as shown by flow cytometry. Subcutaneous injection of tTF-NGR into athymic mice bearing human HT1080 fibrosarcoma tumors induced tumor growth retardation and delay. Contrast enhanced ultrasound detected a decrease in tumor blood flow in vivo after application of tTFNGR. Histological analysis of the tumors revealed vascular disruption due to blood pooling and thrombotic occlusion of tumor vessels. Furthermore, a lack of resistance was shown by re-exposure of tumor-bearing mice to tTF-NGR after regrowth following a first cycle of treatment. However, after subcutaneous (s.c.) push injection with therapeutic doses (1-5 $\mathrm{mg} / \mathrm{kg}$ bw) side effects have been observed, such as skin
\end{abstract}

Correspondence to: Dr Wolfgang E. Berdel or Dr Rolf Mesters, Department of Medicine A, Hematology, Oncology and Pneumology, University of Muenster, Albert-Schweitzer-Strasse 33, D-48129 Muenster, Germany

E-mail: berdel@uni-muenster.de

E-mail: rolf.mesters@ukmuenster.de

${ }^{*}$ Contributed equally

Abbreviations: tTF, truncated tissue factor; NGR, peptide GNGRAHA; VDA, vascular disrupting agent(s); APN, aminopeptidase N; CEUS, contrast enhanced ultrasound

Key words: truncated tissue factor, subcutaneous therapy, vascular targeting, cancer, tumor-homing peptides bleeding and reduced performance. Since lethality started within the therapeutic dose range $\left(\mathrm{LD}_{10}\right.$ approximately $2 \mathrm{mg} / \mathrm{kg}$ bw) no safe therapeutic window could be found. Limiting toxicity was represented by thrombo-embolic events in major organ systems as demonstrated by histology. Thus, subcutaneous injection of tTF-NGR represents an active, but toxic application procedure and compares unfavourably to intravenous infusion.

\section{Introduction}

The formation of new blood vessels is essential for spread and metastasis of solid tumors (1) and is also observed in some hematological malignancies (2). This process assures supply of oxygen and nutrients as well as removal of metabolic waste products (3). Angiogenesis and neovascular endothelial cells have become promising targets for antitumor therapy, as the inhibition of blood supply subsequently should lead to reduction of tumor growth $(3,4)$.

To date, mainly two strategies affecting tumor blood supply have been developed. The first one inhibits angiogenesis by interfering with the process of formation of new blood vessels. Representative drugs of this group, such as the monoclonal antibody against Vascular Endothelial Growth Factor (VEGF) bevacizumab, are already clinically established (5). An alternative approach is to destroy already existing tumor blood vessels by so-called vascular disrupting agents (VDA), such as combretastatins (3). Damaging tumor blood vessels will disrupt both upstream and downstream function with subsequent cell death. In this regard, tumor vasculature is considered as the 'Achilles' heel' of solid tumors. Even a change of shape or function of endothelial cells may be adequate to destabilize the vascular system in tumors.

Direct contact to blood flow makes tumor endothelial cells easy to reach, and Denekamp et al were the first to propose these endothelial cells as a target for antitumor therapy (6). Specific tumor vessel markers such as the vascular endothelial growth factor-receptor 2 (7), endosialin (8), the matrixmetalloproteinases (9), several integrins such as $\alpha_{v} \beta_{3}(10)$, 
$\alpha_{v} \beta_{5}(11), \alpha_{1} \beta_{1}$ and $\alpha_{2} \beta_{1}(12)$, and NG2 proteoglycan (13) and others are promising targets for specific ligands (14-18) coupled with cytotoxic and proapoptotic substances and targeting these effector molecules to tumor endothelial cells. This design of molecules appropriate for vascular targeting is to be conceptually separated from the use of small molecule VDA exploiting specific characteristics of the tumor vasculature and disrupting tumor vessels. Several agents of this latter group such as combretastatins and flavonoids are tested in clinical trials (19).

Here we report on data targeting the tissue factor (TF), a central initiator of the extrinsic coagulation pathway in vivo, to tumor vessels which results in tumor vessel infarction and cell death. The lack of coagulation inducing activity of a soluble form of TF missing its transmembrane domain (truncated TF: tTF) (20) can partly be recovered by relocalizing tTF into the proximity of a phospholipid membrane (21). Accordingly, targeting tTF via peptides and antibodies to specific tumor vessel markers including the MHC complex (21), the ED-B domain of fibronectin (22), the prostate specific antigen (23), the vascular cell adhesion molecule (VCAM-1) (24), and integrins such as $\alpha_{v} \beta_{3}$ (25) has lead to rapid induction of thrombosis in tumor vessels.

Recent studies revealed that small peptides containing the NGR motif (asparagine-glycine-arginine) bind to aminopeptidase N (APN; CD13) (26), a surface molecule with upregulated expression on endothelial cells in tumors and tissues that undergo angiogenesis. NGR binds differentially to CD13 in tumor vasculature, but not to CD13 in normal kidney and myeloid cells (27). In addition, NGR undergoes a rapid nonenzymatic asparagine deamidation to isoaspartate-glycinearginine (isoDGR) generating a further ligand for $\alpha_{v} \beta_{3}(28,29)$. We have constructed fusion proteins consisting of NGRpeptide sequences coupled to the C-terminal end of tTF $(30,31)$. Several of these fusion proteins including tTF-NGR, retain their thrombogenic activity in vitro, bind to their respective targets on endothelial cells, and upon intravenous (i.v.) infusion induce thrombosis in blood vessels in several solid tumors in mice with subsequent tumor growth retardation and regression (30,31). I.v. infusion of tTF-NGR in cancer patients at dose levels without side effects was shown to reduce tumor blood flow in situ (30). In this study, we have analysed antitumor activity and toxicity profile of s.c. injection of tTF-NGR in human tumor xenograft models, s.c. injection of tTF-NGR represents an active but toxic way of application and compares unfavourably to the i.v. infusion of the compound.

\section{Materials and methods}

Cloning, expression, and purification of proteins. The cDNA coding for tTF containing amino acids 1-218 and tTFGNGRAHA, in which the heptapeptide is linked to the Cterminus of tTF (tTF-NGR), was amplified by polymerase chain reaction (PCR) using the primers 5'-CATGCCATGGG ATCAGGCACTACAAATACTGTGGCAGCATATAAT-3' (5'-Primer), 5'-CGGGATCCTATTATGCATGTGCTCTTC CGTTACCTCTGAATTCCCC-3' (3'-Primer) for tTF-NGR. With the DNA-Ligation kit (Novagen, Schwalbach am Taunus, Germany) the cDNA was cloned into the expression vector pET30a(+) (Novagen) using the BamHI and NcoI sites of the vector. The generated tTF constructs possess an $\mathrm{N}$ terminal histidine tag for the subsequent purification of the proteins by using immobilized metal-chelate affinity chromatography.

The vectors were introduced into competent $E$. coli cells (BL21 DE3) according to the manufacturer's protocol (Novagen). After stimulating with IPTG (Novagen), the cells were harvested and 5-7 $\mathrm{ml}$ lysis buffer $(10 \mathrm{mM}$ Tris- $\mathrm{HCl}$, $\mathrm{pH} 7.5 ; 150 \mathrm{mM} \mathrm{NaCl} ; 1 \mathrm{mM} \mathrm{MgCl} ; 10 \mu \mathrm{g} / \mathrm{ml}$ aprotinin; $2 \mathrm{mg} / \mathrm{ml}$ lysozyme) per gram wet weight and $20 \mu \mathrm{l}$ benzonase (Novagen) were added to the pellet. Then the cells were incubated for $90 \mathrm{~min}$ at room temperature (RT) and centrifuged at $12,000 \mathrm{x} \mathrm{g}$ for $20 \mathrm{~min}$ at $4^{\circ} \mathrm{C}$. The pellet was resuspended and homogenized by sonicating in washing buffer $(10 \mathrm{mM}$ Tris/HCl, pH 7.5; 1 mM EDTA (ethylene-diaminetetraacetic acid), 3\% Triton $\mathrm{X}-100)$. To solubilize the inclusion bodies, 2-4 ml guanidinium buffer [ $6 \mathrm{M} \mathrm{GuCl}, 0.5 \mathrm{M} \mathrm{NaCl}, 20 \mathrm{mM}$ Tris/ $\mathrm{HCl}, \mathrm{pH} 7.5$, and $1 \mathrm{mM}$ dithiothreitol (DTT)] per gram wet weight was added. After incubation overnight at RT, the suspension was centrifuged at $10,000 \mathrm{x}$ g for $15 \mathrm{~min}$ at $4^{\circ} \mathrm{C}$. The supernatant was filtered through a $0.22-\mu \mathrm{m}$ filter and stored at $-25^{\circ} \mathrm{C}$. To upscale the manufacturing procedure a multistep HPLC-based purification process has been established (HPLC unit: ÄKTA purifier 100 system, GE Healthcare, Uppsala, Sweden). The first capture step consists of an immobilized metal-affinity chromatography (IMAC) in which the immunogenic nickel is replaced by copper (IMAC Sepharose 6 FF, GE Healthcare). The histidine-tagged (Nterminus) tTF-NGR fusion protein binds to the immobilized copper ions so that the complete refolding (urea gradient from 6 to $0 \mathrm{M}$ ) and washing processes are performed on the column, from which the tTF-NGR protein is eluted by applying $300 \mathrm{mM}$ imidazole. During the subsequent gel filtration the IMAC eluate is conditioned by a buffer exchanging step using Sephadex G-25 (GE Healthcare) in order to prepare for the following intermediate purification step. This anionexchange chromatography step (AIEX; Q Sepharose HP, GE Healthcare) allows further separation of the eluted proteins according to differences in their net charges. Moreover, it removes most of the remaining impurities such as other proteins, nucleic acids and endotoxines. The concluding polishing step again comprises a gel filtration using Sephadex G25 in order to remove any remaining trace impurities and to exchange the buffer to PBS. The final protein solutions are stored at $-80^{\circ} \mathrm{C}$.

The tTF-NGR protein was analyzed under denaturing conditions on SDS-PAGE and Western blotting using mouse monoclonal anti-human tissue factor antibody (American Diagnostica, Pfungstadt, Germany; dilution 1:1000). Besides, every charge was analyzed by the Factor-X coagulation assay. Here, the ability of the tTF-NGR protein to enhance the specific proteolytic activation of FX by FVIIa was evaluated by Michaelis-Menten analyses (32). Analyses of the bacterial endotoxin content of the tTF fusion protein charges have routinely been accomplished by a certified external laboratory (Charles River Laboratories, L'Arbresle, France). The used kinetic chromogenic LAL (Limulus amebocyte lysate) assay was performed in compliance with the European Pharmacopoeia (sensitivity: $0.005 \mathrm{IU} / \mathrm{ml}$ ). The determined values were 
always below an endotoxin level of $10 \mathrm{IU} / \mathrm{mg}$ protein (1.4$7.1 \mathrm{IU} / \mathrm{mg}$ protein). tTF-NGR solutions were micro-filtrated with $22-\mu \mathrm{m}$ filters before use in the in vivo experiments.

Cell culture. Human umbilical vein endothelial cells (HUVECs) were from BD Biosciences. The HT1080 fibrosarcoma cell line (30) was cultured in Dulbecco's medium supplemented with $10 \%$ fetal calf serum (FCS, Gibco-BRL, Eggenstein, Germany).

Binding studies using flow cytometry. HUVECs were used at low passage numbers and grown in serum-supplemented medium. We applied flow cytometry using monoclonal antibodies (AB) against CD13 (BD Biosciences, Heidelberg; cat. no. 347837, PE-linked), $\alpha_{v} \beta_{3}$ (CD51/CD61; BD Pharmingen, Heidelberg, cat. no. 550037, PE-linked) to verify presence of these molecules on HUVECs (details not shown) (30). For binding analysis, cells were blocked using human immunoglobulin ( $\mathrm{Ig}$ ), washed and incubated with His-tag-tTF-NGR for 20 min at RT at different concentrations as specified. Subsequently, cells were washed twice (PBS/ $1 \%$ bovine serum albumin (BSA)/0.1\% $\mathrm{NaN}_{3}$ ) and incubated with either PE-linked anti-CD13 antibody, PE-linked antiCD51/CD61 antibody or isotype control antibody (BD Biosciences) as specified for $30 \mathrm{~min}$ at $37^{\circ} \mathrm{C}$. After 3 further washing steps, cells were directly measured using a FACSCalibur $^{\mathrm{TM}}$ flow cytometer (Becton-Dickinson, San Jose, USA). To show that cells remaining without anti-CD13 or anti-CD51/CD61 binding in these assays had surface-bound His-tag-tTF-NGR present, cells were (after washing) incubated with mouse anti-His-tag (Novagen, Madison, WI, USA, cat. no. 70796) antibody, washed twice, incubated with fluorescein isothiocyanate (FITC)-conjugated goat anti-mouse secondary Ig (BD Biosciences; indirect immunofluorescence), washed $\mathrm{x} 3$, and measured.

Effect of tTF-NGR on apoptosis of endothelial cells in vitro. To test for a putative cell death-inducing potential of the tTFNGR protein after binding to endothelial cells, cytoplasmic histone-associated DNA fragments were determined by the use of the Cell Death Detection ELISA ${ }^{\text {Plus }}$ kit from Roche (Mannheim, Germany) according to the manufacturer's instruction. HUVECs $\left(2.5 \times 10^{4}\right.$ cells $\left./ \mathrm{ml}\right)$ were incubated with different protein concentrations for $18 \mathrm{~h}$. The apoptosis inducer camptothecin was used as positive control.

Effect of tTF-NGR on proliferation of endothelial cells in vitro. To quantify a putative effect on endothelial cell proliferation of tTF-NGR, the incorporation of the thymidine analogue bromodeoxyuridine (BrdU) during the DNA synthesis was measured colorimetrically by the use of the Cell Proliferation ELISA from Roche (Mannheim, Germany) according to the manufacturer's instruction. HUVECs $\left(1 \times 10^{5}\right.$ cells $\left./ \mathrm{ml}\right)$ were incubated with different protein concentrations for $32 \mathrm{~h}$. The subsequent BrdU incorporation was performed for additional $16 \mathrm{~h}$.

Animals. All procedures on animals were performed in agreement with German regulations (Tierversuchsgesetz $\S 8$ Abs. 2) and specifically approved in form of a project license.
Five to seven week-old CD-1 nude mice were purchased from Charles River Laboratories (Sulzfeld, Germany) and acclimated to our animal-experiment facility for at least 1 week before any examination. Mice were maintained on a 12:12-h light:dark cycle in a low-stress enviroment $\left(22^{\circ} \mathrm{C}, 50 \%\right.$ humidity, low noise) and given food and water ad libitum.

Tumor mouse models. The tumor cells for the experiments were grown as attached monolayer cultures, dissociated in trypsin, centrifuged, washed and resuspended in PBS. For evaluation of the antitumor effect, mice were injected s.c. with the HT1080 cells in one rear flank with $4 \times 10^{6}$ cells/mouse in $100 \mu 1$. To estimate the volume of the tumor, its exposed surface was measured in two perpendicular directions and the volume was calculated as (length $\mathrm{x}$ width ${ }^{2} \mathrm{x} \pi / 6$ ). Tumor growth was allowed to a volume of approximately $500-2000 \mathrm{~mm}^{3}$. Different tumor volumes were chosen as starting points for randomization and treatment to study possible tumor growth retarding effects of early and tumor shrinkage of late treatment onset. The mice were randomly assigned to different experimental groups. Saline or experimental drugs were injected s.c. distant from the tumors using various schedules and doses. To study development of resistance, mice with regrowing tumors after a first cycle of treatment were re-exposed to s.c. treatment with tTF-NGR after an observation period without therapy; control mice with tumors at similar size were treated for the first time in parallel.

Histology. Histological analyses were performed on paraformaldehyde fixed (4\%) and paraffin-embedded tissues as described earlier (2). Briefly, tissues embedded in paraffin were cut to $4-\mu \mathrm{m}$ sections and transferred onto glass slides. For identification of thrombosis and organic pathologies, H\&E stained sections were examined using conventional light microscopy in a blinded fashion.

Blood tests. Blood for analysis of coagulation parameters was received by transthoracic puncturing of hearts with animals under anaesthetic. Subsequent determination of PTT (partial thromboplastin time), TT (thrombin time) and Quick-value was accomplished by standard procedures.

Contrast enhanced ultrasound. Contrast enhanced ultrasound (CEUS) was performed using an ultrasound machine dedicated for small animal CEUS (Vevo 2100, Visualsonics, Toronto, Canada) equipped with an $18-\mathrm{MHz}$ transducer operating in a contrast specific imaging mode (powermodulation). A commercially available ultrasound contrast agent was constantly injected via a $27 \mathrm{G}$-tail vein catheter using a rotating infusion pump at $20 \mu \mathrm{l} / \mathrm{min}$ (33). A fixed crosssectional view of the tumor was obtained. The adjacent kidney served as control tissue. Regions of interest were placed either covering the tumor or the kidney during the experiment. Imaging was gated by ECG and respiration to ensure comparable conditions. Steady state of the contrast signal was achieved after $2 \mathrm{~min}$ and signal intensity in the control organ was constant during the experiment. Forty-five micrograms tTF-NGR was push-injected s.c. Start of injection marked (t 0 ) and measurements of linearized signal intensities given in acoustic units (AU) were plotted versus time as previously described (34). 
A 1. His-tTF-NGR $\quad 0.3 \mu \mathrm{g} / \mathrm{mL}$ 2. CD13 PE $5 \mu \mathrm{g} / \mathrm{mL}$
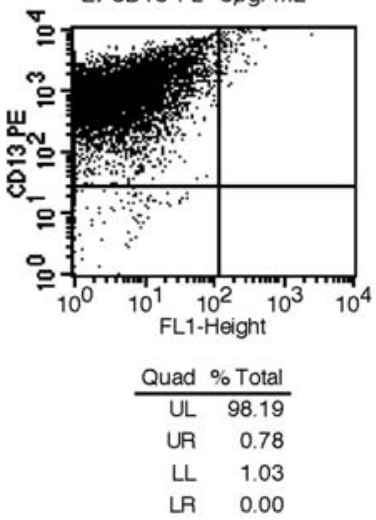

B

1. His-tTF-NGR $0.3 \mu \mathrm{g} / \mathrm{mL}$ 2. $\mathrm{CD} 51 / 61 \mathrm{PE} 3 \mu \mathrm{g} / \mathrm{mL}$
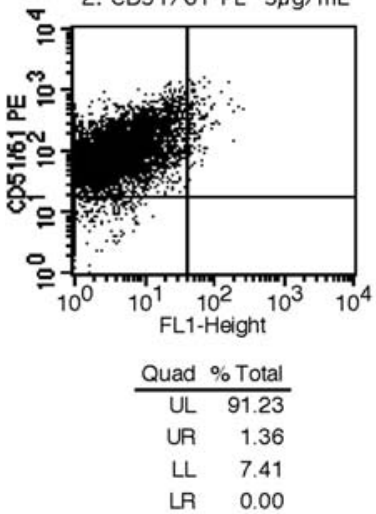

1. His-tTF-NGR $30 \mu \mathrm{g} / \mathrm{mL}$

2. $\mathrm{CD} 13 \mathrm{PE} 5 \mu \mathrm{g} / \mathrm{mL}$

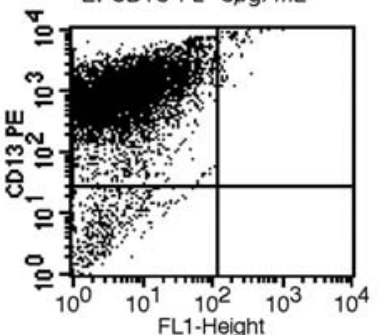

Quad \% Total

UL $\quad 95.23$

UR 0.62

LL $\quad 4.15$

LR $\quad 0.00$

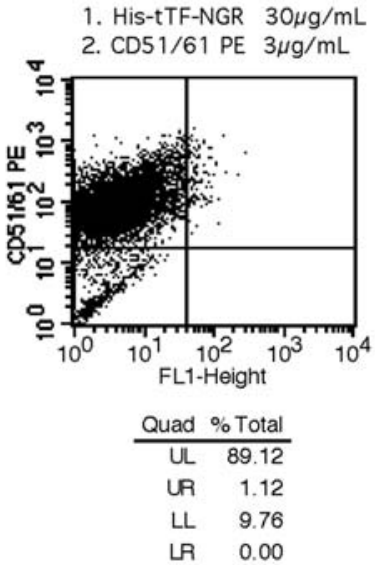

1. His-tTF-NGR $300 \mu \mathrm{g} / \mathrm{mL}$

2. CD13 PE $5 \mu \mathrm{g} / \mathrm{mL}$
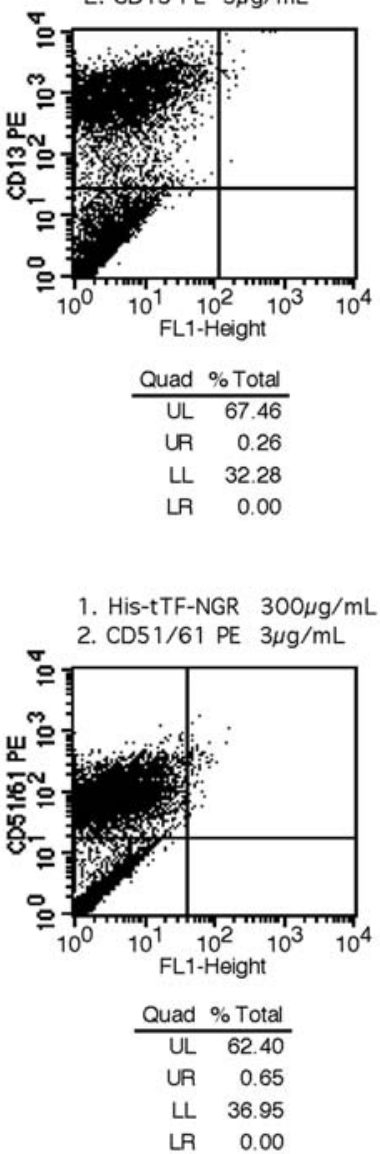

C 1. His-tTF-NGR $300 \mu \mathrm{g} / \mathrm{mL}$

2. $\mathrm{CD} 13 \mathrm{PE} 5 \mu \mathrm{g} / \mathrm{mL}$

3. HisTag mAb $4 \mu \mathrm{g} / \mathrm{mL}$

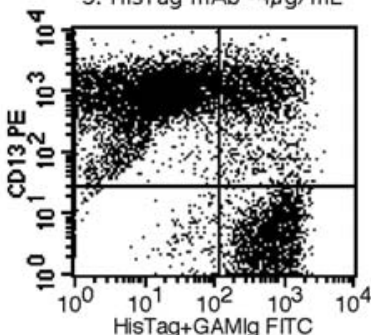

\begin{tabular}{cr} 
Quad & $\%$ Total \\
\hline UL & 66.01 \\
UR & 15.02 \\
LL & 1.98 \\
LR & 16.99
\end{tabular}

D 1. His-tTF-NGR $300 \mu \mathrm{g} / \mathrm{mL}$

2. $\mathrm{CD} 51 / 61 \mathrm{PE} 3 \mu \mathrm{g} / \mathrm{mL}$

3. HisTag mAb $4 \mu \mathrm{g} / \mathrm{mL}$
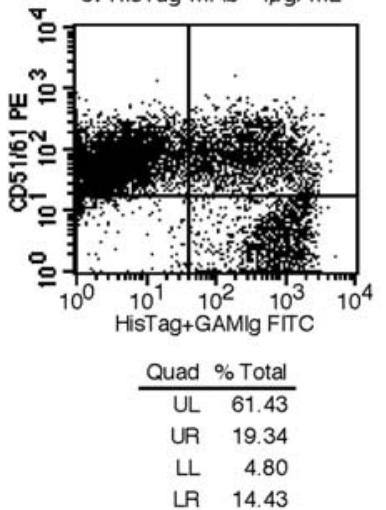

Figure 1. Binding of tTF-NGR (and deamidation products) as measured by flow cytometry. (A) Blocking of anti-CD13 antibody binding by preincubation of HUVECs with increasing concentrations of His-tagged tTF-NGR (UL, upper left; UR, upper right; LL, lower left, representing cells without anti-CD13 antibody binding; LR, lower right). (B) Blocking of anti-CD51/CD61 ( $\left.\alpha_{v} \beta_{3}\right)$ antibody binding by preincubation of HUVECs with increasing concentrations of His-tagged tTF-NGR (and deamidation product). (C and D) Presence of His-tagged tTF-NGR as shown by anti-His antibody binding on HUVECs with (UR) and without (LR) anti-CD13 (C) or anti CD51/CD61 (D) antibody binding.

Statistics. Statistical significance of differences was tested by the Mann-Whitney rank sum test for independent groups. Two-sided $\mathrm{P}<0.05$ were considered significant.

\section{Results}

Binding studies with flow cytometry. To study specific binding of tTF-NGR (and deamidation products) with flow cytometry we first characterized HUVECs as carrying relevant (CD13, $\alpha_{v} \beta_{3}$ ) binding sites (details not shown) (30). Next, we preincubated HUVECs with increasing concentrations of tTF-NGR (0.3-300 $\mu \mathrm{g} / \mathrm{ml}$ His-tag-tTF-NGR) and subsequently measured binding of a constant concentration $(5 \mu \mathrm{g} / \mathrm{ml})$ of either anti-CD13- or anti-CD51/CD61-antibody. As can be seen in Fig. 1A and B (LL) increasing concentrations of tTF-NGR could compete with binding of both antibodies to HUVECs. To further test for presence of tTF-NGR on the cells without anti-CD13- and anti-CD51/CD61-antibody binding we performed subsequent indirect immunofluorescence studies detecting His-tagged tTF-NGR (Fig. 1C and D). These studies indicate specific binding of tTF-NGR and deamidation products to both known binding sites CD13 and $\alpha_{v} \beta_{3}$ and are in agreement with previous binding studies using different protocols for flow cytometry (30).

Effect of s.c. tTF-NGR injections on growth of HT1080 xenotransplants. The antitumor activity of tTF-NGR was determined in CD-1 nude mice bearing HT1080 tumors. Drugs and controls were push-injected s.c. five times at intervals of $24 \mathrm{~h}$. Injections of 3, 5 or $7 \mathrm{mg}$ tTF-NGR $/ \mathrm{kg}$ body weight (bw) induced tumor shrinkage and subsequent growth delay for a longer period of time when compared with saline controls. However, after termination of treatment a regrowth could be observed within some days. S.c. injection of $1 \mathrm{mg} / \mathrm{kg}$ bw seemed to have no major influence on tumor growth when compared with saline controls. Representative results are presented in Fig. 2A. Subsequently, we reduced treatment to two subcutaneous push-injections at intervals of $24 \mathrm{~h}$. This regimen also induced a delay of HT1080 tumor growth (Fig. 2B).

Next, we studied characteristics of tumor growth under prolonged therapy with tTF-NGR. Mice obtained 5 injections in daily intervals and were then randomized to receive 2 further injections with tTF-NGR versus no further treatment. 

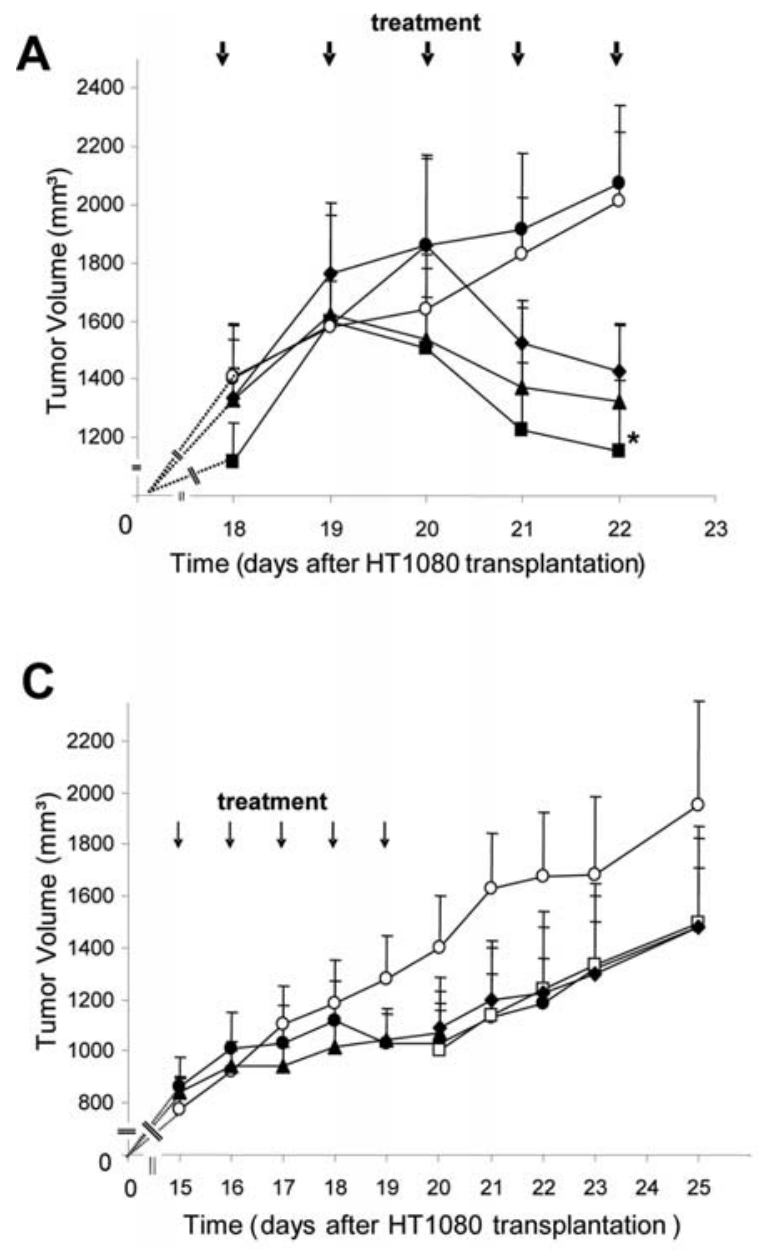
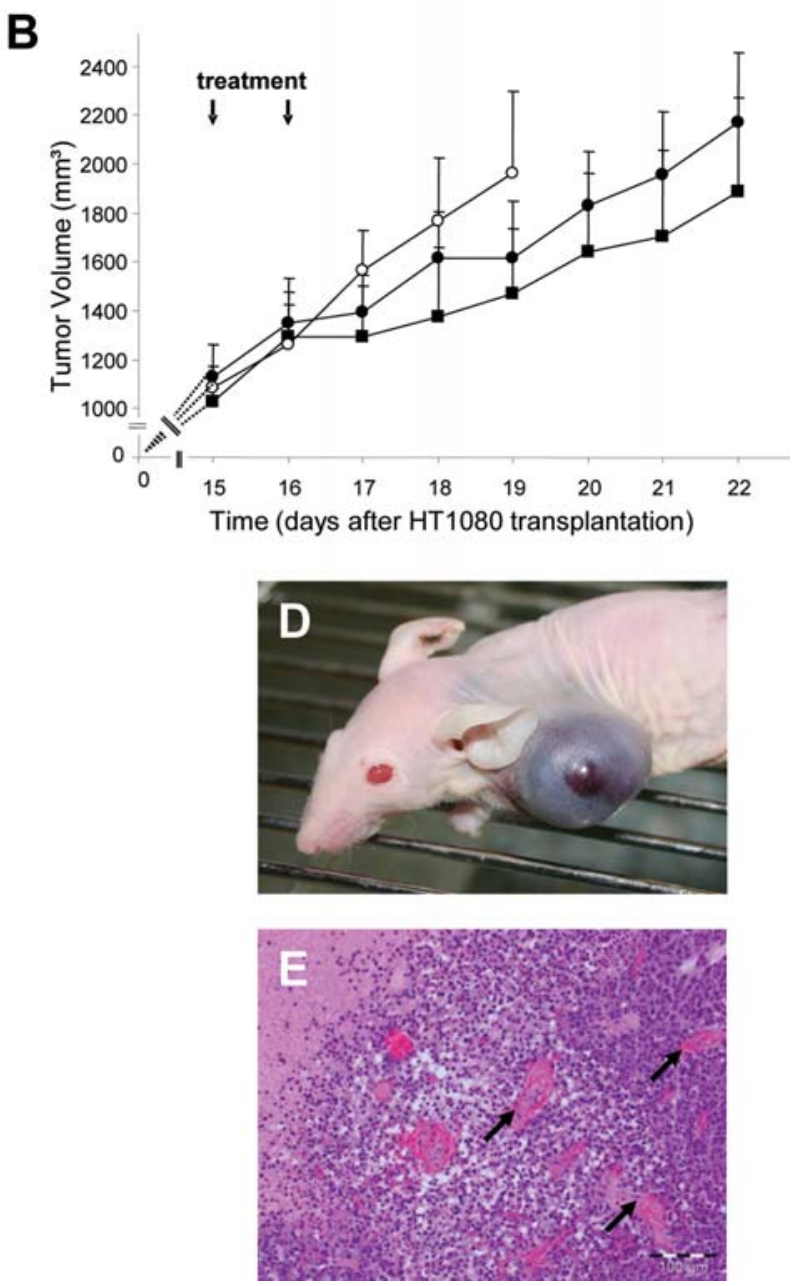

Figure 2. Antitumor activity of s.c. tTF-NGR. (A) Growth inhibition of human HT1080 xenotransplanted into athymic CD-1 mice by s.c. injection of tTF-NGR $[1 \mathrm{mg} / \mathrm{kg}$ bw $(\bullet), \mathrm{n}=8 ; 3 \mathrm{mg} / \mathrm{kg}$ bw $(\boldsymbol{\Delta}), \mathrm{n}=6 ; 5 \mathrm{mg} / \mathrm{kg}$ bw $(\boldsymbol{\bullet}), \mathrm{n}=6 ; 7 \mathrm{mg} / \mathrm{kg}$ bw $(\bullet), \mathrm{n}=7]$ compared to saline [(o), $\mathrm{n}=8]$. Arrows indicate the time points of tTF-NGR injection. Data are presented as means with standard errors. Asterisk denotes statistical significance between tTF-NGR and saline (Mann-Whitney rank-sum test; P<0.05). (B) Growth delay of HT1080 xenotransplants by s.c. injection of tTF-NGR [1 mg/kg bw (•), n=7, or $5 \mathrm{mg} / \mathrm{kg}$ bw ( $\bullet$ ), n=6] compared to saline $[(0), n=6]$. Arrows indicate the time points of injection (2 applications). Data are presented as means with standard errors. (C) Growth inhibition of HT1080 by i.v. [1 mg/kg bw $(\bullet), n=7$ ] and s.c. [3 mg/kg bw $(\mathbf{\wedge}), \mathrm{n}=17$ ] administration of tTF-NGR compared to saline [(o), $\mathrm{n}=6$ ]. Arrows indicate the time points of injection (5 applications). After five s.c. injections, the $3-\mathrm{mg} / \mathrm{kg}$ s.c. group $(\boldsymbol{\Delta})$ was randomized into two subgroups: one received no further therapy [( $\bullet)$, $\mathrm{n}=5]$, the other received two further s.c. injections of tTF-NGR [3 mg/kg bw ( ()$, \mathrm{n}=4]$. (D) Tumor color and necrosis after s.c. injection of tTF-NGR (5 m/kg bw). (E) Occlusion of tumor vessels (arrows, original magnification x200) after s.c. tTF-NGR.

As shown in Fig. 2C, this prolonged application of tTF-NGR could not inhibit tumor regrowth as compared to no further application. In these experiments the activity of tTF-NGR at $3 \mathrm{mg} / \mathrm{kg}$ s.c. was comparable to $1 \mathrm{mg} / \mathrm{kg}$ i.v. indicating a comparably lower antitumor activity achieved by the s.c. route of application.

In the majority of cases, tumors treated with tTF-NGR showed blue and brown coloration and necrotic areas (Fig. 2D). In case of overt ulcerations of the tumor animals were sacrificed and censored. Histology revealed blood pooling, vascular disruption and thrombosis in tumor vessels (Fig. 2E).

Development of resistance. To study development of resistance, mice with regrowing tumors after the first cycle of therapy were re-exposed to s.c. treatment with tTF-NGR after an observation period of $>2$ weeks without tTF-NGR therapy, while control mice with tumors at similar size were treated for the first time in parallel. A lack of resistance development upon re-exposure to tTF-NGR could be shown (Fig. 3A), as HT1080 tumor shrinkage was similar when comparing the effects of 1 st versus 2 nd treatment cycles.

However, the HT1080 xenograft model revealed some variability of tumor growth and treatment effects: besides experiments, in which we observed therapeutic effects of tTFNGR, there were few experiments, in which repeated s.c. injections of $2-5 \mathrm{mg} / \mathrm{kg}$ bw tTF-NGR induced neither shrinkage nor delay of tumor growth (details not shown). In addition, s.c. injections of $3 \mathrm{mg} / \mathrm{kg}$ bw every second day instead of daily injections influenced tumor growth only to a lesser extent (data not shown).

Contrast enhanced ultrasound. To study the mode of action of the in vivo tTF-NGR activity, CEUS imaging was started immediately after injecting $45 \mu \mathrm{g}$ tTF-NGR s.c. Shortly after tTF-NGR injection tissue perfusion decreases firstly in the center and consecutively also in the periphery of the tumor. 

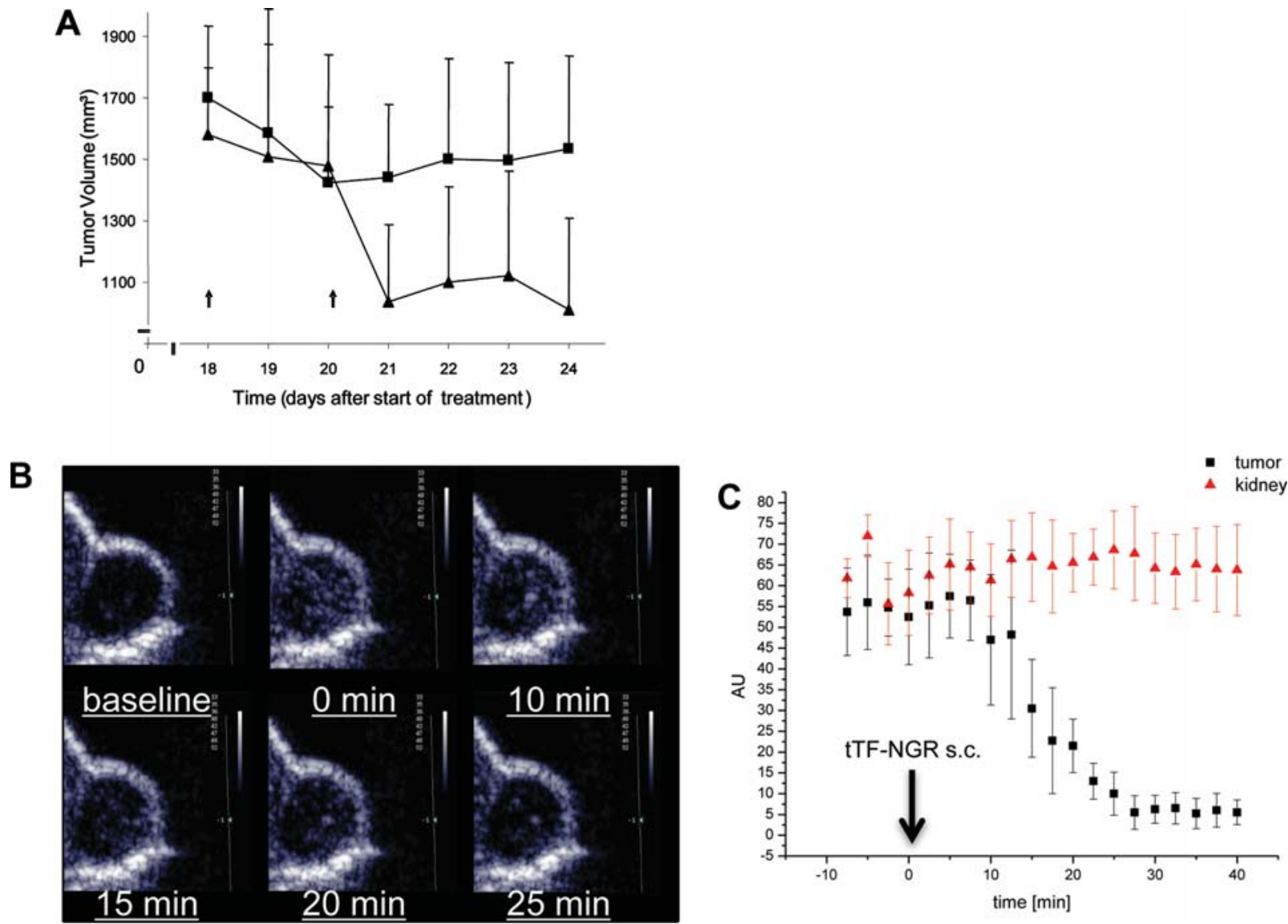

Figure 3. Re-exposure experiments and sonography. (A) Re-exposure of HT1080 xenografts previously treated with tTF-NGR (after tumor re-growth, $\mathbf{\Delta}$ ) in comparison to previously untreated ( $\bullet$ ) xenotransplant-bearing animals. Growth of HT1080 was inhibited by s.c. injection of tTF-NGR (3 mg/kg bw, each group with $n=4)$. Arrows indicate the time points of injection. Data are presented as means with standard errors. There were no significant differences between the groups. (B) Contrast enhanced ultrasound (CEUS) imaging of tumors demonstrates tumor perfusion. Baseline image (left upper) shows nearly no ultrasound signals within the tumor. At steady state of contrast injection (t $0 \mathrm{~min}$ ) s.c. injection of $45 \mu \mathrm{g}$ tTF-NGR was performed. Note homogeneous distribution of CEUS signals prior to tTF-NGR injection. Prior to contrast injection consecutive images demonstrate decrease of contrast enhancement. Note decrease of CEUS signals within the tumor to nearly baseline level. (C) Quantitative analysis of CEUS. Linearized signal intensities given as acoustic units

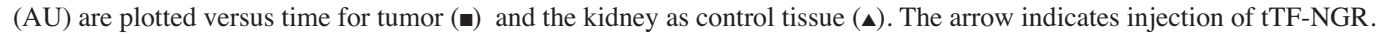

After 15-20 min CEUS depicts a considerable reduction of perfusion signals (Fig. 3B). The measurements of linearized signal intensities given as AU plotted versus time of observation revealed the decrease of tumor blood flow compared to the blood flow of the control organ kidney (Fig. 3C).

Toxicity of s.c. $t T F-N G R$. Acute tolerability of $1-3 \mathrm{mg} / \mathrm{kg}$ bw s.c. was good. Upon s.c. injection of $5 \mathrm{mg} / \mathrm{kg}$ bw, mice suffered from visible weakness and exhaustion. Cutaneous hematomas occurred around the injection site within $24 \mathrm{~h}$ after treatment (Fig. 4A). Infrequently, especially after administration of higher doses ( $>3 \mathrm{mg} / \mathrm{kg} \mathrm{bw}$ ), also distant cutaneous hematomas, e.g. in the face have been observed. Hematomas regressed mostly within one week after termination of treatment. Furthermore, in some mice sudden deaths occurred within $24 \mathrm{~h}$ after the first injection of tTF-NGR with preceding clinical signs of dyspnea. During all experiments with s.c. application of tTF-NGR or saline we evaluated the cumulative incidence of sudden deaths according to dose groups $(1,3,5,7 \mathrm{mg} / \mathrm{kg}$ bw). Cumulative data show an increasing number of deaths within a period until $24 \mathrm{~h}$ after termination of treatment with using higher doses of tTF-NGR. Lethal dose for $10 \%$ of the animals $\left(\mathrm{LD}_{10}\right)$ was found at approximately $2 \mathrm{mg} / \mathrm{kg}$ (Fig. 4F). Mice treated with toxic doses of tTF-NGR (7 mg/kg bw s.c.) and those dying upon treatment were subjected to histopathological examination of all major organ systems. Histopathology revealed peripheral pulmonary embolism (Fig. 4B), necrosis zones in kidneys and liver (Fig. 4C and D) and parietal thrombus in hearts (Fig. 4E). All other organs seemed to be unaffected. To further study possible reasons for cutaneous and organ bleeding with parallel pulmonary embolism, we subjected mice to blood coagulation tests upon receiving high doses of tTF-NGR $(7 \mathrm{mg} / \mathrm{kg}$ bw i.v.). Preliminary results showed parameters compatible with disseminated intravascular coagulation (DIC), as there was elongation of PTT, TT and drop of Quick-value (details not shown).

Analysis of a direct impact of tTF-NGR on vascular endothelial cells. Thrombotic occlusion of tumor blood vessels with the subsequent reduction of tumor blood flow and tumor necrosis has been shown as the main mode of action of the tTF-NGR therapy $(25,30,31)$. In order to exclude a direct effect of tTFNGR on cell death (apoptosis) and on proliferation of vascular 

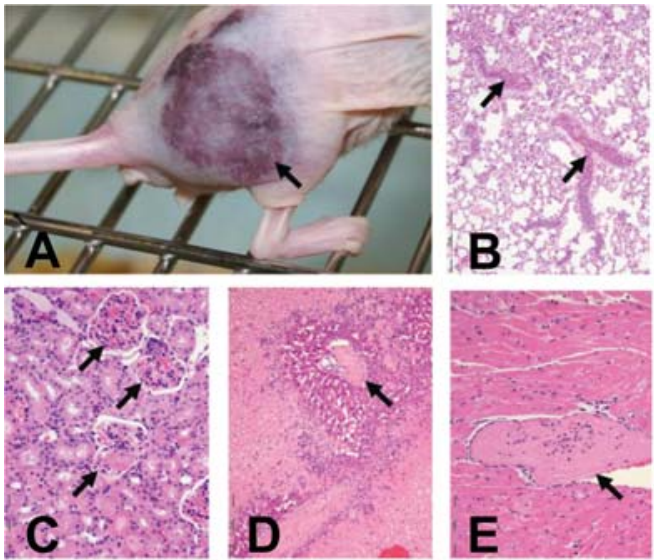

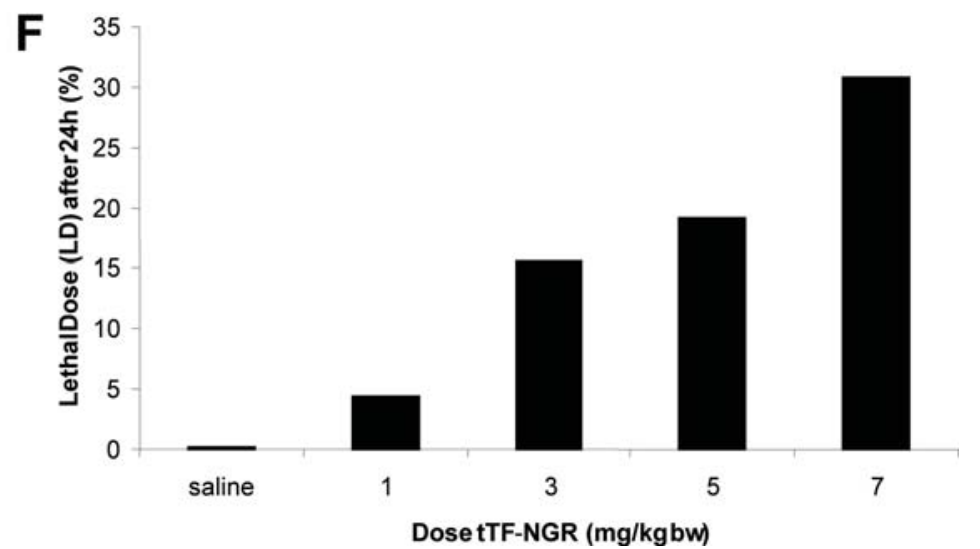

Figure 4. Toxicology studies. (A) Cutaneous hematoma around the injection area $24 \mathrm{~h}$ after treatment. (B-E) H\&E staining of mouse organs after s.c. treatment with tTF-NGR in toxic doses: (B) Peripheral pulmonary embolism (arrows indicating the occluded blood vessels). (C) Tubulus-necrosis and thrombosis (arrows) in the kidney. (D) Necrosis and granulation of liver-tissue, thrombosis in central vein (arrow). (E) Cardiac parietal thrombus (arrow). Original magnification x200 (B and D) and x400 (C and E). (F) Lethality within $24 \mathrm{~h}$ after last s.c. tTF-NGR injection. Spontaneous deaths and mice sacrificed upon intolerable side effects were counted. Cumulative data are given as percentage of all mice in the respective s.c. dose groups with saline $(\mathrm{n}=23), 1(\mathrm{n}=23), 3(\mathrm{n}=26), 5(\mathrm{n}=26)$ and $7(\mathrm{n}=26) \mathrm{mg} / \mathrm{kg}$ bw, respectively.

A

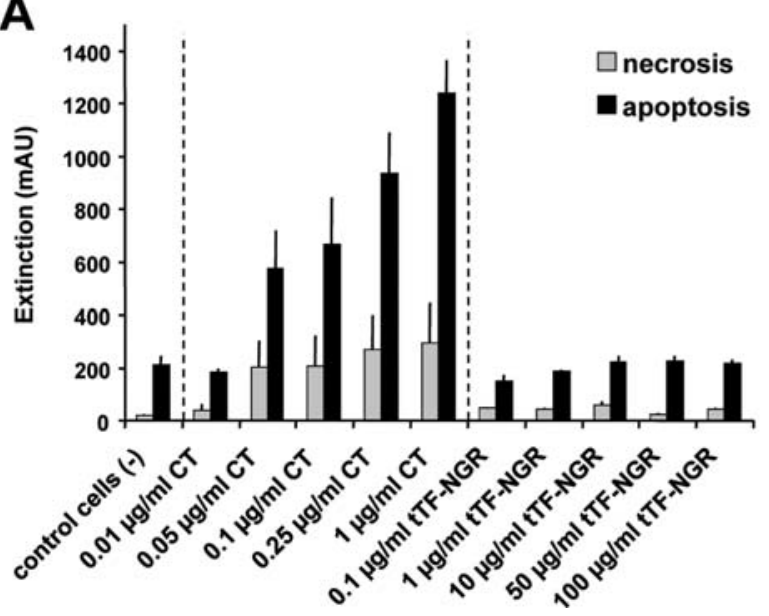

B

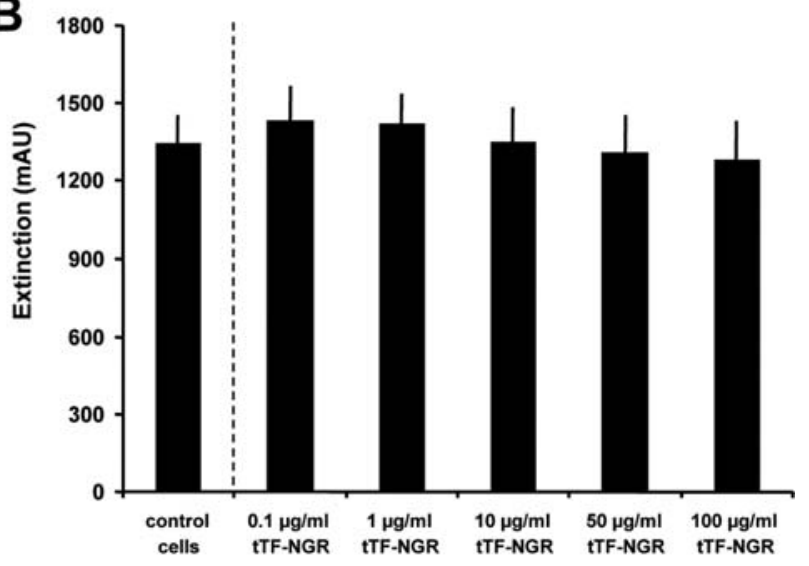

Figure 5. In vitro analysis of tTF-NGR effects on endothelial cell death (A) and proliferation (B), respectively. Human umbilical vein endothelial cells (HUVECs) have been incubated with increasing concentrations of the tTF-NGR protein $(0.1-100 \mu \mathrm{g} / \mathrm{ml})$. To test for cell death-inducing potential, the amount of histone-associated DNA fragments after a $16 \mathrm{~h}$-incubation period has been determined in tTF-NGR-incubated HUVECs in comparison to cells incubated in ascending concentrations $(0.01-1 \mu \mathrm{g} / \mathrm{ml})$ of the apoptosis inducer camptothecin (CT). While CT concentrations greater than $0.05 \mu \mathrm{g} / \mathrm{ml}$ clearly induce cell death, no significant apoptotic effect was detectable after tTF-NGR incubation (A). In a colorimetric immunoassay determining the incorporation of BrdU during DNA synthesis of proliferating cells, increasing tTF-NGR concentrations had no effect on proliferation of HUVECs compared to control cells (B). Data of 2-3 independent assays are presented as means $\pm \mathrm{SE}$.

endothelial cells, ELISA-based assays have been performed (Fig. 5). Neither the viability (Fig. 5A) nor the ability of endothelial cells to proliferate (Fig. 5B) were influenced by tTF-NGR, even at concentrations several-fold higher than those reached in vivo within the therapy experiments (data not shown). We have also assayed tTF-NGR concentrations lower than shown in Fig. 5 with no effects on cell death or proliferation.

\section{Discussion}

There have been a few reports on using antibody-coupled tTF for targeting markers of angiogenesis in tumor vessels inducing thrombosis with subsequent growth reduction and tumor shrinkage $(21,22,24)$. Important limitations of using antibodies as targeting moieties for tTF might be their nonspecific accumulation and uptake in the reticuloendothelial system and the immunogenicity of the resulting antibodyeffector molecule complex. Thus, using antibodies fused to tTF could cause dose-limiting procoagulant activities in liver, spleen and bone marrow, as well as neutralizing antibodies. Furthermore, the bigger size of antibodies might cause sterical hindrance and reduced procoagulant activity of tTF. To avoid described limitations, we expressed a fusion protein consisting of a small peptide (GNGRAHA) coupled to the C-terminus of tTF. 
The NGR-motif was discovered by in vivo screening of phage libraries (11), while tTF is part of the tTF:VIIa complex (35), a potent initiator of coagulation. Via aminopeptidase $\mathrm{N}$ (CD13), to which small peptides containing the NGR motif can bind, the tTF-NGR fusion protein adopts an orientation perpendicular to the phospholipid membrane of the endothelial cell providing a basis for coagulation. By connecting the peptide to the C-terminus of tTF, sterical hindrance of the interaction of tTF, FVII and FX should be eliminated (35). In addition, peptides do not bind to the reticuloendothelial system, are chemically stable and easy to derivatize (36). CD13 is upregulated within tumors (37) and in response to hypoxia and angiogenic growth factors. In addition, the inhibition of the CD13 pathway abrogates endothelial cell migration, invasion and morphogenesis of endothelial cells $(38,39)$. Interestingly, there have been reports on differential binding of NGR-drug conjugates, as they bind to CD13 on tumor endothelial cells but not to CD13 expressed on hematopoietic cells and epithelial cells in proximal tubules in the normal kidney (27). This can be explained by recognition of CD13 isoforms expressed within tumor-associated vessels but not on normal tissues. In addition, higher phosphatidylserine expression within the tumor vasculature might provide additional selectivity for pro-coagulant activities induced by tTF-NGR (27). A further hypothetical advantage of tTF-NGR is that deamidation of the NGR-peptide may generate isoDGR, an $\alpha_{v} \beta_{3}$ ligand which is also upregulated on tumor endothelial cells (28). Therefore, the described mechanism may allow for targeting of two tumor-specific binding sites by one ligand and might amplify procoagulant activity of tTF-NGR. In the results presented here moreover, we could exclude a dominant direct effect of our tTF-NGR construct on viability or proliferation of endothelial cells as a mechanism underlying the in vivo therapeutic effects. Coagulation inducing activity of i.v. application of tTF-NGR in tumor vasculature with subsequent tumor infarction and growth delay could be shown in several tumor mouse models $(30,31)$. The first inman experiences with very low doses of tTF-NGR $\left(1-4 \mathrm{mg} / \mathrm{m}^{2}\right.$ by i.v. infusion) showed inhibition of tumor perfusion without any side effects (30).

Whenever working with E. coli derived material, endotoxin/ LPS contamination might complicate interpretation of the results. Philipp et al (40) used minimal $10 \mathrm{ng}$ total dose LPS ( $1 \mathrm{IU}$ is equivalent to $\sim 0.1 \mathrm{ng}$ ), which was able to induce coagulation on tumor endothelium if co-administered with soluble tissue factor. Here, we used tTF-NGR charges that contained approximately $7.5 \mathrm{pg}$ LPS (refering to $1 \mathrm{mg}$ tTF protein $/ \mathrm{kg}$ bw of a $30 \mathrm{-g}$ mouse) up to approximately $50 \mathrm{pg}$ LPS (refering to $7 \mathrm{mg}$ tTF protein/ $\mathrm{kg}$ bw of a $30-\mathrm{g}$ mouse) per injection, which is 200-1300 times less compared to the administered LPS doses of Philipp et al, excluding endotoxin effects. Furthermore, in previous series of experiments we used non-targeted tTF, produced in the same E. coli-strain using identical protocols as a control substance $(25,30,31)$. Non-targeted tTF has never shown visible antitumor effects or thrombogenic efficacy in vivo, and this also excludes endotoxin effects. Because of the lack of activity of tTF, within this series of experiments, we have used saline instead of tTF as control vehicle.
The investigation reported here was undertaken to test for alternative and potentially easier routes of application. Results show that s.c. injection of tTF-NGR is causing tumor growth delay of HT1080 fibrosarcoma xenografts in vivo. However, doses therapeutically active as i.v. infusions ( $1 \mathrm{mg} / \mathrm{kg} \mathrm{bw})$ (30), had only low or no effect after s.c. injection, and higher doses with antitumor activity occurred together with limiting toxicity not allowing for a safe therapeutic window. Thus, s.c. injection of tTF-NGR represents an active, but toxic application procedure and compares unfavourably to the i.v. infusion. The i.v. infusion of tTF-NGR and analogs alone or in combination with cytotoxic drugs and/or radiation will be further studied as an experimental antitumor therapy.

\section{Acknowledgements}

This study was supported by grants of the Deutsche Krebshilfe e.V. (109245 to W.E. Berdel), the Deutsche Forschungsgemeinschaft (SFB656, projects C8 and C3/Z2), IZKF Muenster (Core unit ECHO), FP7 (SonoDrugs), and the Sybille-HahneStiftung. W.E. Berdel and R.M. Mesters share a patent application on vascular targeting of tissue factor constructs. The other authors declare no competing financial interests.

\section{References}

1. Folkman J: Angiogenesis in cancer, vascular, reumatoid and other disease. Nat Med 1: 27-31, 1995.

2. Padró T, Ruiz S, Bieker R, et al: Increased angiogenesis in the bone marrow of patients with acute myeloid leukemia. Blood 95: 2637-2644, 2000.

3. Chaplin DJ, Dougherty GJ and Siemann DW: Vascular disrupting agents. In: Antiangiogenic Cancer Therapy. Davis DW, Herbst R and Abbruzzese JL (eds). CRC Press, Boca Raton, pp329-364, 2008.

4. Ferrara $\mathrm{N}$ and Alitalo K: Clinical applications of angiogenic growth factors and their inhibitors. Nat Med 5: 1359-1364, 1999.

5. Hurwitz H, Fehrenbacher L, Novotny W, et al: Bevacizumab plus irinotecan, flourouracil, and leucovorin for metastatic colorectal cancer. N Engl J Med 350: 2335-2342, 2004.

6. Denekamp J: Endothelial cell proliferation as a novel approach to targeting tumour therapy. Br J Cancer 45: 136-139, 1982.

7. Kessler T, Fehrmann F, Bieker R, Berdel WE and Mesters RM: Vascular endothelial growth factor and its receptor as drug targets in hematological malignancies. Curr Drug Targets 8: 257-268, 2007.

8. Rettig WJ, Garin-Chesa P, Healey JH, Su SL, Jaffe EA and Old LJ: Identification of endosialin, a cell surface glycoprotein of vascular endothelial cells in human cancer. Proc Natl Acad Sci USA 89: 10832-10836, 1992.

9. Kessler TA, Pfeifer A, Silletti S, et al: Matrix metalloproteinase/integrin interactions as target for anti-angiogenic treatment strategies. Ann Hematol 81 (Suppl 2): S69-S70, 2002.

10. Brooks PC, Clark RA and Cheresh DA: Requirement of vascular Integrin alpha $\mathrm{V}$ beta 3 for angiogenesis. Science 264: 569-571, 1994.

11. Koivunen E, Wang B and Ruoslahti E: Phage libraries displaying cyclic peptides with different ring sizes: ligand specificities of the RGD-directed integrins. Biotechnology (N Y) 13: 265-270, 1995.

12. Senger DR, Claffey KP, Benes JE, Perruzzi CA, Sergiou AP and Detmar M: Angiogenesis promoted by vascular endothelial growth factor: regulation through alpha1beta1 and alpha2beta1 integrins. Proc Natl Acad Sci USA 94: 13612-13617, 1997.

13. Burg MA, Pasqualini R, Arap W, Ruoslahti E and Stallcup WB: NG2 proteoglycan-binding peptides target tumor neovasculature. Cancer Res 59: 2869-2874, 1999.

14. Arap W, Pasqualini R and Ruoslahti E: Cancer treatment by targeted drug delivery to tumor vasculature in a mouse model. Science 279: 377-380, 1998. 
15. Ellerby MH, Arap W, Ellerby LM, et al: Anti-cancer activity of targeted pro-apoptotic peptides. Nat Med 5: 1032-1038, 1999.

16. Hood JD, Bednarski M, Frausto R, et al: Tumor regression by targeted gene delivery to the neovasculature. Science 296 2404-2407, 2002.

17. Ran S, He J, Xianming H, Soares M, Scothorn D and Thorpe PE: Antitumor effects of a monoclonal antibody that binds anionic phospholipids on the surface of tumor blood vessels in mice. Clin Cancer Res 11: 1551-1562, 2005.

18. Ruoslahti E: Targeting tumor vasculature with homing peptides from phage display. Semin Cancer Biol 10: 435-442, 2000.

19. Tozer GM, Kanthou C and Baguley BC: Disrupting tumour blood vessels. Nat Rev Cancer 5: 423-435, 2005.

20. Morrissey JH, Macik BG, Neuenschwander PF and Comp PC: Quantitation of activated factor VII levels in plasma using a tissue factor mutant selectively deficient in promoting factor VII activation. Blood 81: 734-744, 1993.

21. Huang X, Molema G, King S, Watkins L, Edgington TS and Thorpe PE: Tumor infarction in mice by antibody-directed targeting of tissue factor to tumor vasculature. Science 275 : 547-550, 1997.

22. Nilsson F, Kosmehl H, Zardi L and Neri D: Targeted delivery of tissue factor to the ED-B domain of fibronectin, a marker of angiogenesis, mediates the infarction of solid tumors in mice. Cancer Res 61: 711-716, 2001

23. Liu C, Huang H, Donate F, et al: Prostate-specific membrane antigen directed selective thrombotic infarction of tumors. Cancer Res 62: 5470-5475, 2002.

24. Ran S, Gao B, Duffy S, Watkins L, Rote N and Thorpe PE: Infarction of solid Hodgkin's tumors in mice by antibody-directed targeting of tissue factor to tumor vasculature. Cancer Res 58: 4646-4653, 1998.

25. Kessler T, Bieker R, Padró T, et al: Inhibition of tumor growth by RGD peptide-directed delivery of truncated tissue factor to the tumor vasculature. Clin Cancer Res 11: 6317-6324, 2005.

26. Pasqualini R, Koivunen E, Kain R, et al: Aminopeptidase N is a receptor for tumor-homing peptides and a target for inhibiting angiogenesis. Cancer Res 60: 722-727, 2000.

27. Curnis F, Arrigoni G, Sacchi A, et al: Differential binding of drugs containing the NGR motif to CD13 isoforms in tumor vessels, epithelia, and myeloid cells. Cancer Res 62: 867-874, 2002.

28. Corti A, Curnis F, Arap W and Pasqualini R: The neovasculature homing motif NGR: more than meets the eye. Blood 112: 2628-2635, 2008.
29. Curnis F, Longhi R, Crippa L, et al: Sponaneous formation of L-isoaspartate and gain of function in fibronectin. J Biol Chem 281: 36466-36476, 2006.

30. Bieker R, Kessler T, Schwöppe C, et al: Infarction of tumor vessels by NGR-peptide directed targeting of tissue factor. Experimental results and first-in-man experience. Blood 113: 5019-5027, 2009.

31. Kessler T, Schwöppe C, Liersch R, et al: Generation of fusion proteins for selective occlusion of tumor vessels. Curr Drug Discov Technol 5: 1-8, 2008.

32. Ruf W, Rehemtulla A, Morrissey JH and Edgington TS: Phospholipid-independent and -dependent interactions required for tissue factor receptor and cofactor function. J Biol Chem 266: 2158-2166, 1991.

33. Lohmaier S, Ghanem A, Veltmann C, Sommer T, Bruce M and Tiemann K: In vitro and in vivo studies on continuous echocontrast application strategies using SonoVue in a newly developed rotating pump setup. Ultrasound Med Biol 30: 1145$1151,2004$.

34. Tiemann K, Veltmann C, Ghanem A, et al: The impact of emission power on the destruction of echo contrast agents and on the origin of tissue harmonic signals using power pulseinversion imaging. Ultrasound Med Biol 27: 1525-1533, 2001.

35. Banner DW, D'Arcy A, Chène C, et al: The crystal structure of the complex of blood coagulation factor VIIa with soluble tissue factor. Nature 380: 41-46, 1996.

36. Aina OH, Sroka TC, Chen ML and Lam KS: Therapeutic cancer targeting peptides. Biopolymers 66: 184-199, 2002.

37. Pasqualini R and Arap W: Profiling the molecular diversity of blood vessels. Cold Spring Harb Symp Quant Biol 67: 223-225, 2002.

38. Bhagwat S, Lahdenranta J, Giordano R, Arap W, Pasqualini R and Shapiro L: CD13/APN is activated by angiogenic signals and is essential for capillary tube formation. Blood 97: 652-659, 2001.

39. Bhagwat S, Petrovic N, Okamoto Y and Shapiro L: The angiogenic regulator CD13/APN is a transcriptional target of Ras signaling pathways in endothelial morphogenesis. Blood 101: 1818-1826, 2003

40. Philipp J, Dienst A, Unruh M, et al: Soluble tissue factor induces coagulation on tumor endothelial cells in vivo if coadministered with low-dose lipopolysaccharides. Arterioscler Thromb Vasc Biol 23: 905-910, 2003. 